\title{
Biologic Treatment in Glomerular Disease
}

\author{
David Jayne $^{\mathrm{a}}$ Vladimir Tesar ${ }^{\mathrm{b}}$ \\ a Vasculitis and Lupus Clinic, Addenbrooke's Hospital, Cambridge, UK; ${ }^{b}$ Department of Nephrology, \\ 1st Faculty of Medicine, General University Hospital, Charles University, Prague, Czech Republic
}

The classification of glomerular disease has been based almost entirely on renal histology, but an increasing understanding of the pathogenesis is now introducing change relevant to the development of newer therapies. The introduction of corticosteroid and immunosuppressive drugs undoubtedly improved the outcome of patients with many forms of nephrotic syndrome, lupus nephritis and ANCA-associated vasculitis, but this non-specific treatment has been associated with serious short- and long-term toxicity, especially in patients with frequently relapsing disease. During the last 10 years, new biologic (targeted) treatment has expanded our therapeutic armamentarium, especially in refractory, relapsing or intolerant patients with ANCA-associated vasculitis, lupus nephritis and less commonly for other glomerular diseases.

This special topic section of Nephron Clinical Practice provides the reader with a comprehensive overview of the widening area of biologic treatments in an increasing number of glomerular diseases. The first paper [1] returns to the standard immunosuppressive treatment demonstrating its limitations and the current unmet need of, at least, similarly effective and less toxic treatment. To illustrate the close connection between a better understanding of the pathogenesis of the disease and targeted treatment, the papers on the pathogenesis of ANCA-associated vasculitis [2], lupus nephritis [3], the role of anti-PLA2R antibodies [4] in membranous nephropathy and complement in membranoproliferative glomerulonephritis [5] precede the articles dedicated to the treatment of secondary [6-8] and primary glomerulonephritides [9-11]. The final contribution opens new horizons by reviewing the emerging biologic treatments [12].

We hope you enjoy this introduction to the complex area of the pathogenesis and targeted treatment of glomerular disease and that this information will contribute to greater awareness, earlier diagnosis and referral, better treatment and improved outcome for patients with immune-mediated glomerular diseases.

\section{KARGER}

E-Mail karger@karger.com

www.karger.com/nec
(C) 2014 S. Karger AG, Basel

$1660-2110 / 14 / 1284-0203 \$ 39.50 / 0$
Vladimir Tesar

Department of Nephrology, General University Hospital

$\mathrm{U}$ nemocnice 2

CZ-128 08 Prague 2 (Czech Republic)

E-Mail vladimir.tesar@vfn.cz 


\section{References}

-1 Tesar V, Hruskova Z: Limitations of standard immunosuppressive treatment in ANCA-associated vasculitis and lupus nephritis. Nephron Clin Pract 2014;128:205-215.

-2 Sanders JSF, Abdulahad WH, Stegeman CA, Kallenberg CGM: Pathogenesis of antineutrophil cytoplasmic autoantibody-associated vasculitis and potential targets for biologic treatment. Nephron Clin Pract 2014;128:216223.

$>3$ Liu Y, Anders H-J: Lupus nephritis: from pathogenesis to targets for biologic treatment. Nephron Clin Pract 2014;128:224-231. $\checkmark 4$ Ronco P, Debiec H: Anti-phospholipase $\mathrm{A}_{2}$ receptor antibodies and the pathogenesis of membranous nephropathy. Nephron Clin Pract 2014;128:232-237.

5 Popat RJ, Robson MG: Complement and glomerular diseases. Nephron Clin Pract 2014; 128:238-242.

6 Jones RB: Rituximab in the treatment of antineutrophil cytoplasm antibody-associated vasculitis. Nephron Clin Pract 2014;128:243249.

7 Beckwith H, Lightstone L: Rituximab in systemic lupus erythematosus and lupus nephritis. Nephron Clin Pract 2014;128:250-254.

$>8$ Houssiau FA: Biologic therapy in lupus nephritis. Nephron Clin Pract 2014;128:255260.
$>9$ Cravedi P, Remuzzi G, Ruggenenti P: Rituximab in primary membranous nephropathy: first-line therapy, why not? Nephron Clin Pract 2014;128:261-269.

10 Bomback AS: Eculizumab in the treatment of membranoproliferative glomerulonephritis. Nephron Clin Pract 2014;128:270-276.

11 Kronbichler A, Bruchfeld A: Rituximab in adult minimal change disease and focal segmental glomerulosclerosis. Nephron Clin Pract 2014;128:277-282.

12 Karras A, Jayne D: New biologics for glomerular disease on the horizon. Nephron Clin Pract 2014;128:283-291. 